\title{
Instructional Effects of Syntactic Parsing on Chinese College Students' EFL Reading Rates
}

\author{
Xiaokan Chen ${ }^{1}$, Hongmei $\mathrm{Li}^{2}$, Min Gui ${ }^{1}$ \\ ${ }^{1}$ Wuhan University, School of Foreign Languages and Literature, China \\ ${ }^{2}$ The Fourth Primary School in Wuhan Optical Valley, China \\ Correspondence: Min Gui, Wuhan University, School of Foreign Languages and Literature, China.
}

Received: July 22, 2018

doi:10.11114/jets.v6i11.3470
Online Published: September 28, 2018

URL: https://doi.org/10.11114/jets.v6i11.3470

\begin{abstract}
Foreign language reading serves as the fundamental channel for foreign language acquisition and for information gathering. However, English as a Foreign Language (EFL) learners are generally slow in their reading rates. A number of approaches have been proposed to improve reading rates of EFL learners, underpinned by learning theories. From an information processing perspective, building automaticity in lower-level processes is necessary to allow readers to allocate more cognitive resources for higher-level processes. Given the importance of reading rate and the scarcity of related research, the present study intends to explore the relationship between Chinese college students' syntactic parsing ability and their EFL reading rates, and investigate the effects of syntactic parsing instruction on their EFL reading rates. A total of 87 freshmen from two intact classes participated in the present study. They were divided into an experimental group (43) and a control group (44). The experimental group received a 12-week instruction on syntactic parsing ability. A pretest-posttest design was employed to explore the participants' development of syntactic parsing ability and their reading rates. After the training period, a semi-structured interview was conducted among 10 participants of the experimental to explore their attitudes towards the syntactic parsing ability instruction. Two major findings were revealed. First, there was a close relationship between syntactic parsing and reading rate. Second, syntactic parsing instruction may have positive effects on some students' reading rates in the experimental group. Responses in the interview indicated that the participants held positive attitudes towards the syntactic parsing ability training.
\end{abstract}

Keywords: EFL reading, EFL teaching, reading rate, syntactic parsing

\section{Introduction}

EFL learners have been reported to read at a generally low speed, at about 80 to120 words per minute, while fluent L1 readers read at 250 to 300 words per minute (Grabe, 2008). The huge gap may impede their abilities in acquiring information in English, and pose great challenges for EFL learners to excel in international contexts both academically and professionally (Fraser, 2007; Grabe, 2004; Raymond \& Parks, 2002). Chinese college students are also confronted with this dilemma (Fraser, 2007; Luo, 1993).To help these learners achieve fluent reading, according to an information processing paradigm, efficient processing of both lower and higher level reading processes needs to be acquired (Grabe $\&$ Stoller, 2011). Due to limited cognitive resources, suggestions have been made that in order to improve reading rate, instructional guidance should be provided to enhance lower-level processing abilities (e.g., LaBerge \& Samuels, 1974; Stoddard, Valcante, Sindelar, O'Shea, \& Al , 1993; Grabe, 2008). This study intends to investigate the relationship between Chinese college learners' syntactic parsing ability, one of the lower-level processes, and their EFL reading rates, and explore the instructional effects of syntactic parsing on the learners' EFL reading rates.

From an information processing perspective, reading processes comprise lower-level processes, which includes word recognition, syntactic parsing, and semantic proposition formation, and higher-level processes, which consists of text model of comprehension, situation model of reader interpretation, background knowledge use and inferencing, and executive control (e.g., Grabe, 2008; Grabe \& Stoller, 2011; Koda, 2005; Adams, 1994; Perfetti, 1985). The lower and higher processes take place simultaneously and support each other to achieve efficient and successful reading (Stanovich, 2000). As manifested in the Automaticity Theory, to achieve automaticity in reading, which is the effortless processing of information, efficient attribution of cognitive resources among different processes is necessary (LaBerge 
\& Samuels, 1974). Given that lower and higher level processes both require cognitive resources, which is limited, it is suggested that cognitive sources need to be reserved for the more resource-demanding high-level processing (Reynolds, 2000); therefore the lower-level processes need to be automatized.

As a component of the lower-level processes, efficient syntactic parsing contributes to reading rate. It is also referred to as "text segmentation", "sentence segmentation", "chunking abilities", "chunking processes", etc. To avoid ambiguity, only syntactic parsing is used in this article. Syntactic parsing is a process in which words in larger units of structure are processed to extract grammatical information for meaning construction at clause level (Grabe \& Stoller, 2011). It enables readers to identify "phrasal groupings, word ordering information and subordinate and superordinate relations among clauses", and therefore support clause-level meanings (Grabe \& Stoller, 2011, p16). For example, syntactic parsing ability helps readers to process the sentence "Her speech and performance moved the audience" into larger units of structure, i.e., the subject "her speech and performance" and the predicate "moved the audience", rather than individual words. For fluent readers, for example L1 readers, syntactic processing is automatic. They attribute more attention on syntactically meaningful units, but poor readers read word by word or improperly group the phrases (Kuhn \& Stahl, 2003; Nutall, 1996). Though L2 readers have acquired an overt grammatical knowledge, they are unable to parse automatically. If they are to become fluent readers, they need to develop fast and automatic syntactic parsing ability (Urquhart \& Weir, 1998).

Studies in both L1 and L2 context have investigated the effectiveness of syntactic parsing in improving ESL or EFL readers' reading rates, and different findings have been yielded.

Jandreau, Muncer, \& Bever (1986) delivered reading tasks of both normal texts and texts parsed by a computer program among 44 college students. The results showed that the participants read significantly faster when provided with parsed texts than the normal version. LeVasseur, Macaruso, \& Shankweiler (2008) investigated the effects of reading syntactically parsed texts along with another two training conditions, conventional text and word list on 36 second graders' oral reading rates. Although the gains between different training modes were not significant, the participants gained significant increase of reading rates after syntactic parsing training. However, there is no control group for measuring whether the significant increase was a result of normal reading instead of syntactic training; in addition oral reading is different from silent reading; the result of this study may not be generalized to the instructional effects of syntactic parsing in silent reading.

Different from Jandreau et al. (1986) and LeVasseur et al. (2008), several studies in L1 context reported only limited or no effect of syntactic parsing on reading rate development. Stoddard et al (1993) provided both syntactic parsing training and intonation training to 30 fourth and fifth grade students, over a period of 15 days (20 minutes each day). Reading rates were reported to reveal no significant difference between syntactic parsing instruction and intonation training. Repeated reading revealed significant increase in participants' reading rates between one, three, and seven times, yet it is not clear whether the participants have gained their reading rates to a significant level under the influence of parsing cues or repeated reading.

Carver (1970) made a comparison between the reading rates of 60 college mature readers in reading both parsed texts and conventional texts. No significant difference was found between the reading rates of participants in reading parsed texts and conventional texts. The result is quite different from Jandreau et al (1986). Possible explanations for the differentiated findings may be that the parsing criterion used in Carver's study was to some extent arbitrary, and may not present the properly parsed texts.

Research in L2 context is relatively less than in L1 context. Yamashita and Ichikawa (2010) examined the effects of syntactic parsing on the reading rates of 48 Japanese university students, divided into an advanced level and an intermediate level. Four formats of texts, conventional, syntactically parsed, randomly segmented, and word by word, were administered to the participants to measure their reading rates and reading comprehension. The researchers hypothesized that intermediate level participants were more likely to benefit considering the effectiveness of syntactic parsing in reading. Results showed that the reading rates were significantly different between reading word by word texts and the other three formats, but no significant difference was found between reading syntactically parsed format, randomly segmented format, and word by word format in both groups.

Liu and Bever (2002) conducted a similar study in Chinese context, but used different formats from Yamashita and Ichikawa (2010). 280 college students were assigned to two groups, respectively representing higher English proficiency and lower English proficiency. In Liu and Bever, texts were presented in conventional format, syntactically parsed format, and refined parsed format. The refined version is different from the parsed version in that it avoided print segmentation of syntactically meaningful units at line breaks. The study observed no significant difference between formats.

In another study carried out in Chinese EFL context, Lu (2012) categorized texts into four formats, long parsed format, 
short parsed format, conventional format, and word by word format. This classification seems to integrate several previous studies, but the criterion for long parsed and short parsed format is relatively ambiguous. The study revealed no significant difference in reading rates between long or short parsed format and conventional format, but the difference between long parsed format and short parsed format reached significance level. It is indicated that since texts in short parsed format consist of only a few words in one group, and were presented on computer screen in isolation to other groups, it limited the perceptual span of the participant and impeded reading rates (McConkie \& Rayner, 1975).

Although syntactic parsing ability is conceived as an essential skill for L1 and L2 readers, its impacts on reading rate were not pronounced in several previous studies. Among these studies, only a few have employed instructional treatment, which is necessary for participants to acquire automatic syntactic parsing ability. It is suggested that sufficient training might be necessary in investigating the effects of syntactic parsing on EFL readers' reading rates. Drawing on theoretical and empirical knowledge, two questions are proposed in the present study:

1) What is the relationship between Chinese college students' syntactic parsing ability and their EFL reading rates?

2) What are the effects of syntactic parsing instruction on Chinese college students' EFL reading rates?

\section{Method}

\subsection{Participants}

Participants of the present study were 87 university freshmen enrolled in two intact classes at a key university in China. There were 43 students in the experimental group and 44 students in the control group. Their age ranged from 18 to 20 years old. The experimental group consisted of 3 males and 40 females majoring in Law, German language, Russian language, while the control group was composed of 8 males and 36 females majoring in French Language and Japanese Language.

\subsection{Instruments}

\subsubsection{Measurement of Syntactic Parsing Ability}

Material for measuring syntactic parsing ability consists of 100 items, carefully selected from New College English Grammar (3 $3^{\text {rd }}$ edition) (Zhang, 2012), New College English (Book 3), New Horizon English (Book 2), Oxford Bookworms Series, Cambridge English Readers Series by the authors of the present study. Half of the items are used in the pretest, the other half saved for the posttest. Each set of the items encompasses 16 simple sentences, 16 compound sentences, and 18 complex sentences. A simple sentence in this study is an independent clause with a subject and a predicate; a compound sentence involves at least two independent clauses connected by conjunctions or punctuations; a complex sentence has a main clause and at least one subordinate clause.

Drawing on previous research (Stoddard et al., 1993; Yamashita \& Ichikawa, 2010), the syntactic parsing criterion for the present study follows five principles: 1) the subject and the predicate of a clause are separated. Predicate refers to a major constituent of sentence structure in a binary analysis in which all obligatory constituents other than the subject were considered together. For example, in the sentence "John broke the glass", the subject is "John", and the predicate is "broke the glass"; 2) conjunctions of clauses (e.g., "and", "so", "when") are separated; 3) connective devices consisting of multiple words (e.g., "that is" and "for example") are marked as phrasal groups; 4) noun and verb modifiers are combined with their noun and verb; 5) prepositional and adverbial phrases are separated from the rest of the sentence.

The participants were asked to parse the items with the principles above using slashes. For example, the sentence "Women's role change influences some aspects of social life" should be grouped as "Women's role change/ influences some aspects of social life." If participants parsed the sentence as "Women's role/ change/ influences some aspects of social life", it is assumed that they failed to recognize phrasal groupings in a meaningful manner. A correctly parsed item receives 1 point, summing up to a maximum of 50 points for each set of the tests.

\subsubsection{Measurement of Reading Rate and Reading Comprehension}

The reading material used to measure reading rate and reading comprehension were six passages chosen from the CET 4 test (College English test, Band 4), a standardized test of English language proficiency widely used in China. They are divided into 2 parallel sets ( 3 passages for the pretest, and 3 for the posttest). Each set contains 2 short passages, 300-400 words, and a long passage, about 800 words. The 3 passages for the pretest and 3 for posttest are comparable in terms of word counts, readability, and vocabulary frequency.

Reading rates were obtained by calculating the total number of words of the 3 passages divided by reading time, reported as wpm (words per minute). Total word counts were obtained through Microsoft Word, and reading time was recorded by a software timer. 
Rapid reading without adequate comprehension is meaningless; therefore reading comprehension is measured in the present study. Each passage is followed by several comprehension questions, designed to examine participants' understanding of the main idea rather than details. Each short passage was followed by 5 questions, and each long passage followed by 10 questions, adding up to 20 questions for each set of the tests. Reading comprehension is presented as percentage of correct answers. The current study set $70 \%$ of accuracy rate as the cutoff value for measuring reading rates as in several previous studies (Carver, 1990; Segalowitz \& Hébert, 1990; Nation, 2005).

\subsection{Procedures}

\subsubsection{Pilot Study}

Sixteen freshmen from the same university voluntarily participated in the pilot study. They were introduced the parsing rules and guided by the researchers to complete several practices, and then asked to complete the 100 items. After that, they were asked to read the six passages and complete the questions.

Results showed that the reliability of the items in pretest and posttest were generally high, with Cronbach's Alpha reliability reaching 0.744 in the pretest $(\mathrm{k}=50)$ and 0.776 in the posttest $(\mathrm{k}=50)$. In addition, the differences of minimum scores, maximum scores and mean scores between the pretest and posttest were within 2 points. As for the reliability of reading rate and reading comprehension measurement, strong correlation between the pretest and posttest was revealed respectively in reading rates $(\mathrm{r}=.886, \mathrm{p}<0.01)$ and reading comprehension $(\mathrm{r}=.821, \mathrm{p}<0.01)$. It can be suggested from the pilot study that the measurement for syntactic parsing ability, reading rate, and reading comprehension is reliable.

\subsubsection{Pretest}

The pretest is composed of syntactic parsing ability pretest, reading rates pretest, and reading comprehension pretest. Both the experimental group and the control group completed the reading rates and reading comprehension pretest, but only the experimental group took the syntactic parsing ability pretest. In week 5 , the first session, both the experimental group and the control group were administered the reading rates and reading comprehension pretest. The participants of the experimental group first completed the syntactic parsing ability pretest, and then the reading rates and comprehension pretest. They were provided with the parsing rules and parsing exercises for the simple, compound, and complex sentences. After the participants have become acquainted with the test format, the syntactic parsing pretest was administered to the participants, which included 50 items. After that, they completed the reading and comprehension questions of the pretest material, which was made up of three passages with 20 comprehension questions in total. A software time was displayed on a projection screen for the participants to record their own reading time. When completing comprehension questions, they are asked not to refer to the passages. This requirement was assumed to keep the participants from skimming or scanning, propelling them to read each passage completely in order to elicit their normal reading rates and achieve a balance of reading rate and reading comprehension.

\subsubsection{Syntactic Parsing Instruction}

Instructional material for the experimental group was made up of two parts: 1) teaching material (A New English Grammar (Zhang, 2012); 2) exercise material. The material was selected and adapted from A New English Grammar (Zhang, 2012), New College English (Book 2), New Horizon English (Book 2), College English Integrated Course (Book 2), Reading A-Z Leveled Books.

Instructions of syntactic parsing were incorporated into normal teaching curriculum from week 6 to week 17, a total of 12 sessions. From week 6 to week 7, lectures were given concerning sentence structures of three sentences types and the method of parsing. Each instruction session lasted for 15 minutes. From week 8 to week 17, the participants practiced syntactic parsing skill in class with the exercise material. This practice phase aimed at developing the students' syntactic parsing ability by exercising and consolidating what they have learned. During this phase, they were assigned with a large amount of sentences, paragraphs, and passages to practice their syntactic parsing skill, under the guidance of the lecturer. For example, they were asked to find out 3 simple sentences, 3 compound sentences, and 3 complex sentences from the textbooks, and parse the sentences. After completing the parsing task, they were invited to share their answers with the class. Apart from in-class exercise, they were also assigned syntactic parsing exercise as part of their weekly homework, which is similar to the tasks they did in class.

\subsubsection{Posttest}

The posttest was administered in week 18. The same procedure was employed as in the posttest. Both the experimental group and control group took the tests, but only the experimental group took the syntactic parsing ability posttest. The reading rates and comprehension posttest contained the same number of passages and comprehension questions as in the pretest; the syntactic parsing ability posttest involves the same number of items to parse as in the pretest. The procedures of the experimental phase are summarized in Table 1. 
Table 1. The procedure of the experimental phases

\begin{tabular}{lllll}
\hline Group & Week 5 & Weeks 6-7 & Weeks 8-17 & Week 18 \\
\hline Experimental & Pretest & Syntactic parsing & Practice of syntactic parsing & Posttest \\
& SP, RR, & instruction & skill in class and after class & SP, RR, RC \\
& RC & Normal instruction & Normal instruction & \\
\hline Control & Pretest & Normal instruction & Normal instruction & Posttest \\
& RR, RC & & & RR, RC \\
\hline
\end{tabular}

Note: $\mathrm{SP}=$ syntactic parsing, $\mathrm{RR}=$ reading rate, $\mathrm{RC}=$ reading comprehension

\subsubsection{Semi-Structured Interview}

A semi-structured interview was conducted among participants of the experimental group after the posttest to explore how they perceive syntactic parsing instruction and their understanding of reading rates. Criteria sampling method was employed to select suitable participants for the semi-structured interview. Ten participants were chosen to participate in the interview based on their reading rates change from pretest to posttest. Specifically, 5 out of 10 students have achieved greater gains in reading rates than the average gains, i.e., $21 \mathrm{wpm} ; 3$ participants increased their reading rates at approximately the average level; 2 students, however, only obtained marginal gains, below the average level. The interview was conducted in Chinese for the purpose of acquiring accurate and detailed information. Their responses were audio recorded and transcribed by the researchers. The participants were asked to answer the following 4 pre-determined open-ended questions with their permission:

1) What is your attitude towards syntactic parsing instruction?

2) What are the benefits (if any) you obtained from the instruction?

3) What are the challenges you encountered during the instruction?

4) What are your suggestions on the instruction?

\section{Results}

This section will first present the results of the participants' syntactic parsing ability, reading rates and reading comprehension in the pretest and posttest. Second, participants' responses to the semi-structured interview will be presented concerning their attitudes towards syntactic parsing instruction.

\subsection{Results of Syntactic Parsing Ability Test in Pretest and Posttest}

Only participants in the experimental group took the syntactic parsing ability pretest and posttest. 34 out of the 43 participants' scores were included in the analysis of the pretest, given that 9 out of 43 failed to achieve $70 \%$ correctness in the reading comprehension pretest. The mean score of the 34 participants in syntactic parsing pretest is 38 (out of 50), reaching $76 \%$ correctness of the test. Similarly, statistics of 4 out of the 43 in the experimental group were eliminated from posttest data for failing to answer at least $70 \%$ questions correctly in the posttest. The mean score of the 39 participants in posttest is 47 , about $94 \%$ correctness, reporting a $24 \%$ gain in correctness from the pretest. To further examine the difference of syntactic parsing ability improvement between the pretest and the posttest, an independent t-test was performed in SPSS 23.0.

Table 2. $T$-test statistics of syntactic parsing between pretest and posttest

\begin{tabular}{|c|c|c|c|c|c|c|}
\hline & \multicolumn{3}{|c|}{ Levene's test for equality of variances } & \multicolumn{3}{|c|}{$T$-test for equality of means } \\
\hline & $\mathrm{F}$ & Sig. & $t$ & $d f$ & Sig.(2-tailed) & Mean difference \\
\hline $\begin{array}{l}\text { Equal } \\
\text { variances } \\
\text { assumed }\end{array}$ & 13.361 & .000 & -10.956 & 71 & .000 & -8.105 \\
\hline
\end{tabular}

As shown in Table 2, a significant difference in syntactic parsing ability was found between the syntactic parsing ability pretest and the posttest $(\mathrm{p}=.000<0.05)$.

\subsection{Results of Reading Rates in Pretest and Posttest}

Both the experimental group and the control group completed the reading rates pretest and posttest. The same cutoff value for reading comprehension correctness $(\geqslant 70 \%)$ was applied to the data collected, and therefore the reading rates of 34 out of 43 participants in the experimental group, and 35 out of 44 in the control group was included in the pretest data. Applying the same criterion to posttest data, the reading rates of 39 out of 43 in the experimental group and 37 out of 44 in the control group were reported. The reading rates of both groups in the pretest and posttest are shown in Table 3. 
Table 3. Average reading rates of the experimental group and control group

\begin{tabular}{lll}
\hline Group & Pretest reading rates(wpm) & Posttest reading rates (wpm) \\
\hline Experimental group & 130 & 133 \\
Control group & 151 & 138 \\
\hline
\end{tabular}

In the reading rate pretest, the average reading rate of the experimental group is $130 \mathrm{wpm}$, and $133 \mathrm{wpm}$ for the average reading rate of the control group. An independent t-test was employed to identify whether the participants' reading rates were comparable before the instruction. No significant difference was observed between the groups $(p=.493>0.05)$. The statistics from the reading rate posttest revealed quite different patterns between the two groups. The average reading rate of the experimental group is $151 \mathrm{wpm}$, while the average reading rate of the control group is $138 \mathrm{wpm}$, yielding a significant difference in average reading rate between groups $(\mathrm{p}=.023<0.05)$.

\subsection{Correlation Between Syntactic Parsing Ability and Reading Rate}

A correlation coefficient was computed to examine the relationship between syntactic parsing ability and reading rates both in the pretest and posttest. Only the statistics of the experimental group were used in the analysis of correlation between syntactic parsing ability and reading ability. The scores of several participants were removed from the analysis due to their performance in reading comprehension (failing to correctly answer $70 \%$ or more of the comprehension questions). Applying the $70 \%$ correct answer cut-off value, 34 participants in the pretest were included in the analysis and 39 in the posttest were included in the analysis.

Table 4. Correlation between syntactic parsing ability and reading rate in the pretest

\begin{tabular}{lll}
\hline & Syntactic parsing ability & Reading rate \\
\hline Pearson correlation & 1 & $.705^{* *}$ \\
Sig. (two- tailed) & & .000 \\
$\mathrm{~N}$ & 34 & 34 \\
\hline
\end{tabular}

Note: $* *$ indicates that the correlation is significant at 0.01 level (2-tailed)

Table 5. Correlation between syntactic parsing ability and reading rate in the posttest

\begin{tabular}{lll}
\hline & Syntactic parsing ability & Reading rate \\
\hline Pearson correlation & 1 & $.802^{* *}$ \\
Sig. (two- tailed) & & .000 \\
$\mathrm{~N}$ & 39 & 39 \\
\hline
\end{tabular}

Note: $* *$ indicates that the correlation is significant at 0.01 level (2-tailed)

Statistics for pretest and posttest are shown respectively in Table 4 and Table 5. For the pretest, the correlation coefficient between syntactic parsing ability and reading rate in the pretest was .705 , which was significant at the .01 level. In other words, there is a remarkably strong correlation between syntactic parsing ability and reading rate. For the posttest, the correlation coefficient between syntactic parsing ability and reading rate in the posttest was .802, significant at the .01 level, which again indicates a remarkably high correlation between syntactic parsing ability and reading rate.

\subsection{Results of the Semi-Structured Interview}

The researchers of the present study transcribed the recording and analyzed transcripts by following a series of qualitative steps. First, the participants' oral recordings were transcribed into the written reports. Second, the researchers of the study read through all the participants' responses and eliminated irrelevant information. Third, the themes of each participant's response were classified, and similar themes were categorized under the same theme and translated into English. Fourth, the frequency of each theme was calculated to identify repeated major themes. The four questions are demonstrated as follows.

1) What is your attitude towards the syntactic parsing instruction?

On the whole, all 10 students expressed positive attitudes towards the syntactic parsing ability training method. Although they were furnished with adequate grammatical knowledge and a large quantity of vocabularies, they were still overwhelmed by long complex sentences and fail to draw the meanings at a fast speed. Half of them even reported having to read time and time again to understand a long complex sentence. Their positive responses to the syntactic parsing ability instruction, as well as their remarkable improvement in reading rates revealed in the quantitative analysis, indicate the positive effects of the syntactic parsing ability training.

2) What are the benefits (if any) you obtained from the instruction?

Nine out of 10 students reported increased reading rates through the training. In addition, 8 out of 10 students responded that they were more efficient and faster in dealing with long complex sentences. Besides, half of them highlighted the 
facilitative effects of the training in helping them better locate the key points of a text. Moreover, 2 out of 10 students expressed that they had become more confident in reading relatively long complex texts.

3) What are the challenges you encountered during syntactic parsing instruction?

Three out of 10 students reported demand for more exercise in the training sessions. Besides, 3 students responded that they have grasped syntactic parsing ability, but not to a skilled and highly-efficient degree. Moreover, 3 out of 10 participants reflected that they were relatively fluent and efficient in the use of syntactic parsing ability during the training periods, but may not be able to recall and use the skills when encountering difficult reading texts after class, which indicates that the syntactic parsing ability may have not been automatized, and more practice might be necessary to facilitate more automatized syntactic parsing skills. In addition, 2 out of 10 students considered the instruction to be relatively demanding, and expected more feedback from the instructor.

4) What are your suggestions on syntactic parsing instruction?

Four out of 10 students recommended that there should be more training and should include more difficult material. The training time for the present study was 15 minutes each session. It is suggested that the time amount could be increased to allow more training and practice in class. Furthermore, 2 students advised that material for training and practicing should be more diversified. Besides, 2 students expected more individualized feedback from the instructor.

\section{Discussion}

This section addresses each research question by interpreting research results with reference to relevant theories and empirical studies; furthermore, interpretations of the interview will be presented.

Research question one: What is the relationship between Chinese college students' syntactic parsing ability and their EFL reading rates?

As illustrated in 3.3, correlations between syntactic parsing ability and reading rates in both the pretest $(\mathrm{r}=.705)$ and posttest $(\mathrm{r}=.802)$ was strong. The high correlation in both pretest and posttest revealed that there might be a strong positive relationship between the participants' syntactic parsing ability and reading rates. Examinations of experimental group's reading rates from the pretest to the posttest show that as the syntactic parsing ability scores increased from the pretest to posttest, the participants' reading rates also increased, demonstrating the positive correlation reported above.

This result provides empirical evidence for the automaticity effects of syntactic parsing ability on reading rates. Although the contribution of lower-level processes in fluent reading have been well established theoretically by plenty of researchers (e.g., LaBerge \& Samuels, 1974; Stanovich, 2000), there is a dearth of clear evidence from empirical data. Furthermore, previous studies mainly reported the association of lower-level processes and reading utilizing one measure, i.e., word recognition abilities (e.g., Segalowitz, Segalowitz, \& Wood, 1998; Burrows, 2016). The present finding might provide a strong support for the contribution of syntactic parsing ability to reading rate.

The present result is consistent with two theories: Automaticity Theory and Attentional Resource Emancipation, which suggest the role of automaticity of lower-level processes in enabling readers to allocate much greater attention to more demanding and complicated process, i.e., comprehension (LaBerge \& Samuels, 1974; Reynolds, 2000). Through syntactic parsing instruction, students have adequately increased their reading rates without decreasing their reading comprehension.

Research question two: What are the effects of syntactic parsing instruction on Chinese college students' EFL reading rates?

Before the syntactic instruction, the average reading rate of the experimental group was $130 \mathrm{wpm}$, and $133 \mathrm{wpm}$ for the average reading rate of the control group. No significant difference was found between the groups ( $\mathrm{p}=.493>0.05)$. After the instruction, significant differences were identified between the experimental group and the control group ( $\mathrm{t}=2.324$, $\mathrm{p}=.023<0.05)$. The students in the experimental group increased their reading rates from $130 \mathrm{wpm}$ to $151 \mathrm{wpm}$, about $16 \%$ improvement. While the reading rates of the control group grew from $133 \mathrm{wpm}$ to $138 \mathrm{wpm}$, only a gain of $5 \mathrm{wpm}$, only $4 \%$ progress. The results indicated that integrating syntactic parsing instruction into normal teaching curriculum may improve some the participants' reading rates to a significant degree.

Nevertheless, the finding of significant reading rates increase was inconsistent with Liu and Bever (2002). They found that syntactically parsed texts imposed the strongest impact on students' reading rates, but not to the significant degree. Likewise, the results of the present study are contradictory to the result reported by Yamashita \& Ichikawa's (2010), in which facilitative and direct role of parsing on readers' reading rates were not detected. A primary reason for this inconsistency might be that the participants in the present study received a 12-week long instruction and practice, while Liu and Bever (2002) and Yamashita \& Ichikawa (2010) only employed syntactically parsed texts as a testing method to test students' reading rates, failing to develop more automatized syntactic parsing ability. 
In addition, the report of Chinese college students' EFL reading rates (around 130wpm) before the instruction is consistent with Yamashita \& Ichikawa (2010), with advanced Japanese EFL learners reading conventional texts at $133 \mathrm{wpm}$ and reading parsed texts at $131 \mathrm{wpm}$. The result of reading rate pretest is reminiscent of the previous studies that there exists a fairly huge gap between L1 and L2 reading rates. Specifically, fluent L1 readers normally read at between $250 \mathrm{wpm}$ to $300 \mathrm{wpm}$ without effort or difficulty, whereas L2 readers read at between $80 \mathrm{wpm}$ to $120 \mathrm{wpm}$. Participants in the current study read at an average rate of around $130 \mathrm{wpm}$, with the maximum rate at $203 \mathrm{wpm}$ and the minimum rate at $89 \mathrm{wpm}$. The findings show that even the fastest participants' reading rate is slower than the average reading rate of $\mathrm{L} 1$ readers.

Apart from the quantitative results, participants' responses can also provide insights into the effect of the syntactic parsing ability instruction. As shown in 3.4, the participants generally recognized the effectiveness of the syntactic parsing ability training. Below are some excerpts from the participants in the interview, demonstrating positive attitudes towards the syntactic parsing instruction and the benefits they gained from the training.

"I think syntactic parsing ability is useful for me."

"I bet I have improved my reading rate through the instruction."

"The instruction seems magical, because I forget a variety of sentence patterns, such as attribute clause or object clause. I just try to increase my processing speed of sentences by identifying key constituents and finding their relationship as fast as possible."

"In the past, I didn't know what to read when confronted with long complex sentences in reading. Now guided by syntactic parsing instruction, I am able to identify key constituents of sentences, and focus on the main information. It is beneficial for improving my reading rate as well as other language skills."

"I used to be afraid of long complex sentences, but now no longer restricted by various sentence patterns. I have made much progress in dealing with them. Besides, I become a little more confident in reading different kinds of sentences."

These perceived benefits correspond with several previous observations (Jandeau et al., 1986; Stoddard et al., 1993; Yamashita \& Ichikawa, 2010). The training enables readers to process information quickly and to expand their knowledge of syntax, enabling them to concentrate on key information and main ideas of sentences, and increase their reading rates. Furthermore, the instruction increased the participants' confidence in reading English texts, especially helpful in reading long complex texts.

To summarize, both quantitative and qualitative data of the current study reveal a facilitative influence of the syntactic parsing ability training on Chinese college students' EFL reading rates. The significant increase in reading rate offers additional support to the Automaticity Theory and Attentional Resource Emancipation, and provides empirical evidence for the significant role of automatized syntactic parsing ability in the improvement of EFL learners' reading rates.

\section{Conclusion and Implications}

Noticing the gap between L1 and L2 readers' reading rates and a dearth of research on the effect of syntactic parsing ability instruction on learners' reading rates in China, this study investigates the possible relationship between learners' syntactic parsing ability and their EFL reading rates, and explores the effects of a 12-week instruction of syntactic parsing ability on Chinese college students. In addition, the present study also examines the participants' attitudes towards the training. Two major findings are uncovered.

First, a strong correlation between Chinese college students' syntactic parsing ability and their EFL reading rates were observed. The correlation coefficient between the two variables in the pretest was .705, implying that instruction of syntactic parsing abilities might be necessary to facilitate reading rate. The results of the posttest also revealed a high correlation $(\mathrm{r}=.802)$ between them, echoing the findings in the pretest and indicating the syntactic parsing ability training might possibly improve the EFL learners' reading rates.

Second, a significant difference in reading rates between the experimental group and the control group suggests that the training produced facilitative effects on Chinese college students' EFL reading rates, providing support for the assumption made in the correlation analysis. The results of the current study showed that syntactic parsing instruction improved some participants' reading rates. Significant differences in reading rates change were found between the experimental group and the control group $(\mathrm{t}=2.324, \mathrm{p}=0.023<0.05)$, though effect size was medium (Cohen's $\mathrm{d}=0.52$ ). The average reading rate of the experimental group increased from $130 \mathrm{wpm}$ to $151 \mathrm{wpm}$, with a gain of $21 \mathrm{wpm}$; while the average reading rate of the control group moved from $133 \mathrm{wpm}$ to $138 \mathrm{wpm}$, with a gain of $5 \mathrm{wpm}$.

Third, the current study also revealed the participants' positive attitudes towards the syntactic parsing ability training. All students (10) receiving the interview acknowledge the instructional effects of the training; some mentioned benefits in increased reading rates, ability and confidence in reading long complex texts. Despite the helpfulness of the syntactic 
parsing ability training, some interviewees also reported limited use of syntactic parsing ability such as the difficulty of transferring it to real tasks outside the classroom. This indicates that the participants may need more extensive training to become more automatized in their syntactic parsing skills, which is consistent with the participants' suggestion for more training in the interview.

The current study might contribute to the field of ESL and EFL research and teaching on reading ability by raising both teachers' and students' awareness of the importance of reading rate. Reading comprehension has long been underscored in L2 reading education, while underestimating the role of reading rate. It is expected that this study may encourage teachers to implement reading rate instruction in their classroom teaching. The findings in this study are also expected to enrich teachers' understanding of the effects of syntactic parsing ability on EFL learners' reading rates. Furthermore, it is hoped that this study would provide an empirical evidence for the necessity of syntactic parsing ability training on ESL and EFL learners.

Limitations of the present study should also be addressed for the evaluation of this study and for future studies. Some participants reported that the difficulty levels of exercise should be increased, whereas some noted that the instructional materials were a little difficult for them. It is suggested that future research might incorporate material from a wider range of difficulty, and studies on the effects of syntactic parsing training on participants from groups of differentiated English proficiency could also be a research focus. Besides, the instruction in this study lasted for 12 weeks, $15 \mathrm{~min}$ for each week, which, as reported in the interview with the participants, might not be long enough. Future research could expand the training period to provide participants with more sufficient practice of syntactic parsing ability to facilitate the automaticity of the skill. Furthermore, future studies could involve a larger population from more diversified disciplines, and control for a more balanced gender ratio.

\section{References}

Adams, M. J. (1994). Modeling the connections between word recognition and reading. In R. Ruddell, M. Ruddell, \& H. Singer (Eds.), Theoretical models and processes of reading (4th ed.) (pp. 838-950). Newark, DE: International Reading Association.

Burrows, L., \& Holsworth, M. (2016). The effects of explicit word recognition training on Japanese EFL learners. Reading Matrix: An International Online Journal, 16(2), 81-97.

Carver, R. P. (1970). Effect of "chunked" typography on reading rate and comprehension. Journal of Applied Psychology, 54(3), 288-296. https://doi.org/10.1037/h0029266

Carver, R. P. (1990). Reading rate: A review of research and theory. San Diego, CA: Academic Press.

Fraser, C. (2007). Reading rate in L1 Mandarin Chinese and L2 English across five reading tasks. The Modern Language Journal, 91, 372-394. https://doi.org/10.1111/j.1540-4781.2007.00587.x

Grabe, W. (2004). Research on teaching reading. ARAL, 24, 44-69. https://doi.org/10.1017/S0267190504000030

Grabe, W. (2008). Reading in a second language: Moving from theory to practice. New York, NY: Cambridge University Press. https://doi.org/10.1017/CBO9781139150484

Grabe, W., \& Stoller, F. L. (2011). Teaching and researching reading. Essex, England: Pearson Education.

Jandreau, S. M., Muncer, S. J., \& Bever, T. G. (1986). Improving the readability of text with automatic phrase-sensitive formating. British Journal of Educational Technology, 17(2), 128-133. https://doi.org/10.1111/j.1467-8535.1986.tb00502.x

Koda, K. (2005). Insights into second language reading. New York, NY: Cambridge University Press. https://doi.org/10.1017/CBO9781139524841

Kuhn, M., \& Stahl, S. (2003). Fluency: A review of developmental and remedial practices. Educational Psychology, 95, 3-21. https://doi.org/10.1037/0022-0663.95.1.3

LaBerge, D., \& Samuels, S. J. (1974). Toward a theory of automatic information processing in reading. Cognitive Psychology, 6, 293-323. https://doi.org/10.1016/0010-0285(74)90015-2

LeVasseur, V. M., Macaruso, P., \& Shankweiler, D. (2008). Promoting gains in reading fluency: A comparison of three approaches. Reading and Writing: An Interdisciplinary Journal, 21, 205-230. https://doi.org/10.1007/s11145-007-9070-1

Liu, Z. Q., \& Bever, T. G. (2002). An experimental study of the function of syntactic analysis in reading comprehension. Foreign Language Teaching and Research (bimonthly), 34(3), 219-224.

$\mathrm{Lu}, \mathrm{Y}$. (2012). Effects of lexical chunking and vocabulary size on reading fluency of L2 readers (Master's thesis, 
Nanjing University of Technology, Nanjing, China). Retrieved from http://cdmd.cnki.com.cn/Article/CDMD-10291-1013364401.htm

Luo, X. H. (1993). Enforcement of speed-reading to English Majors. Modern Foreign Languages, (4), 24-28.

McConkie, G. W., \& Rayner, K. (1975). The span of the effective stimulus during a fixation in reading. Perception \& Psychophysics, 17, 578-586. https://doi.org/10.3758/BF03203972

Nation, I. S. P. (2005). Reading faster. PASAA, 36, 21-35.

Nuttall, C. (1996). Teaching reading skills in a foreign language (2nd Ed.). Oxford: Heinemann.

Perfetti, C. A. (1985). Reading ability. New York: Oxford University Press.

Raymond, P., \& Parks, S. (2002). Transitions: Orienting to reading and writing assignments in EAP and MBA contexts. Canadian Modern Language Review, 59, 152-180. https://doi.org/10.3138/cmlr.59.1.152

Reynolds, R. E. (2000). Attentional resource emancipation: Toward understanding the interaction of word identification and comprehension processes in reading. Scientific Studies of Reading, 4(3), 169-195. https://doi.org/10.1207/S1532799XSSR0403_1

Segalowitz, N., \& Hébert, M. (1990). Phonological recoding in the first and second language reading of skilled bilinguals. Language Learning, 40(4), 503-538. https://doi.org/10.1111/j.1467-1770.1990.tb00604.x

Segalowitz, S., Segalowitz, N., \& Wood, A. (1998). Assessing the development of automaticity in second language word recognition. Applied Psycholinguistics, 19, 53-67. https://doi.org/10.1017/S0142716400010572

Stanovich, K. (2000). Progress in understanding reading: Scientific foundations and new frontiers. New York, NY: Guilford Press.

Stoddard, K., Valcante, G., Sindelar, P., O'Shea, L., \& Al, E. (1993). Increasing reading rate and comprehension: the effects of repeated readings, sentence segmentation, and intonation training. Reading Research \& Instruction, 32(4), 53-65. https://doi.org/10.1080/19388079309558133

Urquhart, S., \& Weir, C. (1998). Reading in a second language: Process, product and practice. New York: Longman.

Yamashita, J., \& Ichikawa, S. (2010). Examining reading fluency in a foreign language: effects of text segmentation on L2 readers. Reading in a Foreign Language, 22, 263-283.

Zhang, Z. B. (2012). A new English grammar coursebook (5 $5^{\text {th }} e d$.). Shanghai: Shanghai Foreign Language Education Press.

\section{Copyrights}

Copyright for this article is retained by the author(s), with first publication rights granted to the journal.

This is an open-access article distributed under the terms and conditions of the Creative Commons Attribution license which permits unrestricted use, distribution, and reproduction in any medium, provided the original work is properly cited. 